\title{
Arteria oftálmica en preeclampsia
}

\section{Ophtalmic artery in preeclampsia}

Pablo Santillan ${ }^{1, *}$

a preeclampsia $(\mathrm{PE})$ es un síndrome multisistémico complejo que clásicamente se ha descrito por elevación de la ten-

_sión arterial (sistólica> 140 mmHg y diastólica > 90 mmHg) acompañada de proteinuria en la segunda mitad del embarazo, estos hallazgos se revierten hasta en 6 semanas después del parto[1],[2]. Sin embargo, en la práctica clínica su presentación es muy variable, sus características no son específicas y sus parámetros diagnósticos cuantitativos son arbitrarios por lo que es importante establecer una definición fisiopatológica para mejorar el diagnóstico[3].

Cerca de medio millón de mujeres muere cada año a causa de complicaciones asociadas al embarazo, de las cuales $99 \%$ son de países subdesarrollados[4]. La incidencia de trastornos hipertensivos en la gestación (hipertensión gestacional, PE, eclampsia) ha aumentado en las últimas dos décadas, de una tasa de 57,3 por 1.000 partos hospitalarios en 1994 a 86,5 por 1.000 partos hospitalarios en el 2013[1]. Se estima que la PE complica $5 \%$ a $8 \%$ de embarazos[5]. A nivel global la PE y eclampsia son responsables de $10 \%$ a $15 \%$ de las muertes maternas[6].

Hay dos tipos de PE, la temprana que se presenta antes de la semana 34 que esta ocasionada por una placentación defectuosa y la tardía que se da como resultado de la interacción entre la senectud placentaria y la predisposición materna genética a enfermedades metabólicas y cardiovasculares[3]. Respecto al origen cardiovascular de la PE no se ha elucidado si la insuficiencia cardiaca es causa o consecuencia de una placentación anormal[7].

En la PE temprana hay un deterioro del proceso de angiogénesis placentaria secundario a una respuesta inflamatoria aumentada y a la presencia de estrés oxidativo local, lo que impide una adecuada invasión del trofoblasto y remodelación de las arterias espirales uterinas[8]. Se produce daño endotelial ocasionado por un desbalance entre factores angiogénicos y antiangiogénicos[5].

Fisiopatológicamente la PE es un trastorno placentario caracterizado por la presencia de aumento de la resistencia vascular sistémica, estado de hipercoagulabilidad y disfunción endotelial[2].

El único tratamiento disponible es la terminación del embarazo, lo cual puede aumentar la morbilidad y mortalidad neonatal en ciertos casos[9]. Por esta razón la estrategia principal apunta al tamizaje y prevención. Para la predicción se han estudiado varios factores ya que con las características mater- nas tan solo se identifican $30 \%$ de casos de PE[10]. Por lo que actualmente se recurre a bioquímicos y ecográficos maternos como factores predictores y al uso profiláctico de ácido acetil salicílico[11].

Para predicción ecográfica de PE se usa el índice de pulsatilidad (IP) de las arterias uterinas (AUt) entre las 11 a $13+6$ semanas de gestación, con una sensibilidad de 47,8\% (95\% IC, $39,0 \%-56,8 \%$ ) y una especificidad de $92,1 \%$ (95\% IC, $88,6 \%$ $94,6 \%)[12]-[14]$. El IP AUt se encuentra elevado en mujeres que desarrollarán $\mathrm{PE}$, lo que se observa de manera más marcada en PE de inicio temprano (PE en < 34 SG) o asociada a fetos pequeños para edad gestacional (SGA)[10],[15].

Se han investigado otros factores que anteceden a la PE como la presencia de insuficiencia cardiaca diastólica asintomática demostrada en ecocardiografía, que se asocia a PE de instauración temprana[16]. También se han estudiado potenciales factores predictores como índices Doppler de vasos periféricos, entre los cuales destaca el Doppler de arteria oftálmica (AO). Este tiene varias ventajas como facilidad de medición con un equipo de ultrasonido estándar, sus resultados no se afectan por el tejido adiposo y sus índices tienen rangos de referencia constantes en el embarazo. Por lo mencionado se considera que el Doppler de la AO es aplicable como modelo predictivo en lugares de bajos recursos, con un equipo ecográfico limitado o mal cumplimiento del seguimiento prenatal[17].

Para la valoración Doppler de la AO se requiere que la paciente esté en reposo por 10 minutos en posición supina, con los ojos cerrados. Se realiza con el transductor lineal de 6 a 15 $\mathrm{MHz}$ en sentido transversal sobre el párpado superior previa aplicación de gel de conducción (Figura 1).

La identificación de la AO se realiza mediante aplicación de Doppler color, la AO se encuentra a $15 \mathrm{~mm}$ del disco óptico, superior y medial a la banda hipoecogénica que representa al nervio óptico. Una vez identificada la AO se aplica Doppler pulsado en un volumen de muestra de $2 \mathrm{~mm}$, con un ángulo de isoniación inferior a 20 grados, con una profundidad de 3,0 $4,5 \mathrm{~cm}$, un filtro de $50 \mathrm{~Hz}[10]$.

Se obtienen al menos tres ondas consecutivas similares y se determinan automáticamente los siguientes valores[10],[18]:

- Índice de pulsatilidad (IP).

- Índice de resistencia (IR).

- Primera velocidad sistólica máxima (PVS O PS1).

- Segunda velocidad sistólica máxima (SVS).

- Velocidad diastólica máxima (VDM o PD1). 


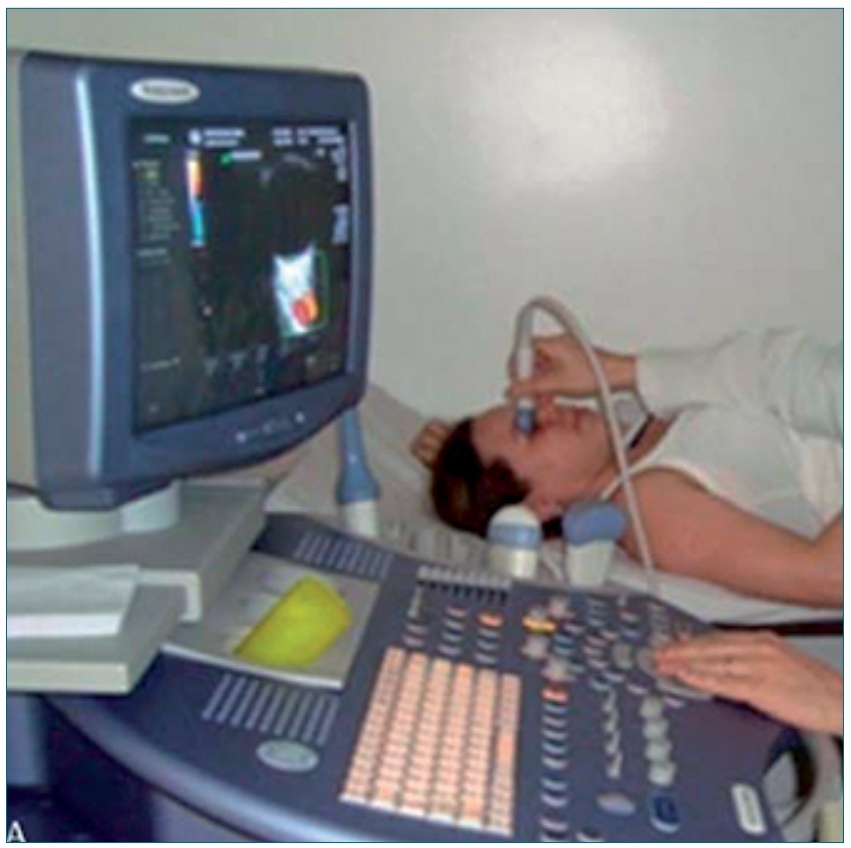

Figura 1. Mujer gestante en posición adecuada para evaluar los parámetros Doppler de la $\mathrm{AO}[10]$.

- Índice de SVS/PVS.

- Índice PS1/PD1.

Se han hecho varios estudios para identificar el parámetro Doppler más apropiado para la arteria oftálmica, se ha observado que PVS tiene una sensibilidad de $61,0 \%(95 \% \mathrm{Cl}$, $44,2 \%-76,1 \%$ ) y especificidad de $73,2 \%$ (95\% IC, 66,9 $78,7 \%$ ) por lo que su efectividad es similar al IP AUt en el primer trimestre[12]. Se ha visto que la aplicación del Doppler de arteria ofttálmica en segundo trimestre no aumenta de manera significativa la detección de PE[10]. Un estudio realizado por Mucize et al. determinó que el IR de la OA es la variable que predice de mejor manera los resultados maternos y la edad gestacional al nacimiento al usar un punto de corte de 0,72, con una sensibilidad y especificidad de $76 \%$ y $76 \%$ respectivamente[19].

El Doppler de las arterias oftálmicas también se ha estudiado como indicador de el estado hemodinámico intracraneal. En la PE hay un estado de hipervascularización cerebral que causa edema. Un estudio valoró parámetros como el IP, IR e índice PS1/PD1; antes y después de la administración de sulfato de magnesio en pacientes con PE severa de presentación temprana. Se evidenció reducción de la perfusión cerebral manifestada como aumento de IP e IR y disminución del índice índice PS1/ PD1 tras la administración del medicamento, lo cual explicaría el mecanismo de acción[20].

Se ha investigado también el uso del Doppler de la AO en la predicción de PE en semana 35 a 37 de gestación, evidenciando que el índice SVS/PVS puede predecir la presentación de PE tardía, especialmente 3 semanas después de la valoración recomiendan usar el promedio de las medidas de ambos ojos para disminuir la variabilidad de los resultados[18] (Figura 2).

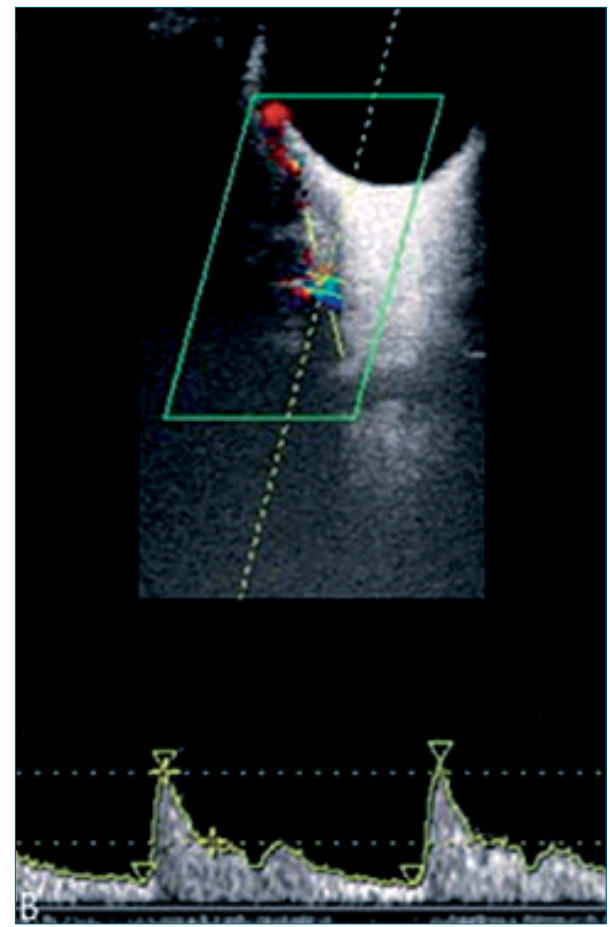

Figura 2. AO identificada al Doppler color, se evalúa a $15 \mathrm{~mm}$ del disco óptico, medialmente al nervio óptico.

\section{Conclusiones}

Tradicionalmente, se ha indicado a la placenta como el origen de la PE, sin embargo, se ha propuesto que este síndrome también podría tener un origen cardiovascular, lo que se evidencia con parámetros distintos a los relacionados con la placentación como el Doppler de las arterias oftálmicas[17].

El estudio Doppler de las arterias oftálmicas tiene sensibilidad y especificidad similares a índice de pulsatilidad de las arterias uterinas, tiene buena reproductibilidad, es aplicable en medios con recursos limitados, no se ve afectado por elementos anatómicos como el tejido adiposo o el útero grávido, por lo que es una buena alternativa como predictor de PE de instauración temprana[17].

\section{Referencias}

1. Harper L. Alan Tita AK. Pregnancy-Related Hypertension. In: Creasy and Resnik's Maternal-Fetal Medicine: Principles and Practice [Internet]. Eighth Edi. Elsevier Inc.; 2019. p. 810-38. Available from: https://doi.org/10.1016/B978-0-323-47910-3.00048-6

2. Onwudiegwu C, Adekanmi A, Olusanya B, Lawal O, Adedokun B, Morhason-Bello I, et al. Imran Morhason-Bello AO. Case-control study on ocular changes and ophthalmic Doppler velocimetric indices among preeclamptic and normotensive pregnant women in Ibadan, Nigeria. BMJ Open Ophthalmol. 2020;5(1):1-7. https:// doi.org/10.1136/bmjophth-2020-000550.

3. Graham J Burton, Christopher W Redman, James M Roberts AM. Pre-eclampsia: pathophysiology and clinical implications. theBMJ. 2019;1-15. 
4. Duley L. The global impact of pre-eclampsia and eclampsia [Internet]. Semin Perinatol. 2009 Jun;33(3):130-7. https://doi. org/10.1053/j.semperi.2009.02.010 PMID:19464502

5. Leaños-Miranda A, Navarro-Romero CS, Sillas-Pardo LJ, RamírezValenzuela KL, Isordia-Salas I, Jiménez-Trejo LM. Soluble Endoglin As a Marker for Preeclampsia, Its Severity, and the Occurrence of Adverse Outcomes. Hypertension. 2019 Oct;74(4):991-7. https://doi.org/10.1161/HYPERTENSIONAHA.119.13348 PMID:31446801

6. Annelien C. De Kat, Jane Hirst, Mark Woodward, Stephen Kennedy SAP. Prediction models for preeclampsia: A systematic review. BMJ Open Ophthalmol. 2019;16:48-66.

7. Melchiorre K, Sutherland GR, Liberati M, Bhide A, Thilaganathan $B$. Prevalence of maternal cardiac defects in women with highresistance uterine artery Doppler indices. Ultrasound Obstet Gynecol. 2011 Mar;37(3):310-6. https://doi.org/10.1002/uog.8850 PMID:20882562

8. Aquino LO, Leite HV, Cabral AC, Brandão AH. Doppler flowmetry of ophthalmic arteries for prediction of pre-eclampsia. Rev Assoc Med Bras. 2014 Nov-Dec;60(6):538-41. https://doi. org/10.1590/1806-9282.60.06.011 PMID:25650853

9. Hastie R, Bergman L, Cluver CA, Wikman A, Hannan NJ, Walker SP, et al. Stephen Tong SH. Proton Pump Inhibitors and Preeclampsia Risk Among 157720 Women. Hypertension. 2019;73(5):1097-103. https://doi.org/10.1161/HYPERTENSIONAHA.118.12547.

10. Praciano de Souza PC, Gurgel Alves JA, Bezerra Maia e Holanda Moura S, Araujo Júnior E. W. P. Martins FDSC. Second Trimester Screening of Preeclampsia Using Maternal Characteristics and Uterine and Ophthalmic Artery Doppler. Ultraschall Med. 2016;x:39.

11. Daniel L. Rolnik, M.D., David Wright, Ph.D., Liona C. Poon, M.D., Neil O'Gorman, M.D., Argyro Syngelaki, Ph.D., Catalina de Paco Matallana, M.D., Ranjit Akolekar, M.D., Simona Cicero, M.D., Deepa Janga, Mandeep Singh, Francisca S. Molina, Walter Plasencia KHN. Aspirin versus Placebo in Pregnancies at High Risk for Preterm Preeclampsia. N Engl J Med. 2017;377:613-22.

12. Velauthar MN. Plana, M. Kalidindi, J. Zamora, B. Thilaganathan, S. E. Illanes, K. S. Khan JA. First-trimester utreine artery Doppler and adverse pregnancy outcome: a meta-analysis involving 55974 women. Ultrasound Obstet Gynecol. 2013
Nov;2014(43):500-7.

13. Khalil A, Cowans NJ, Spencer K, Goichman S, Meiri H, Harrington K. First-trimester markers for the prediction of pre-eclampsia in women with a-priori high risk. Ultrasound Obstet Gynecol. 2010 Jun;35(6):671-9. https://doi.org/10.1002/uog.7559 PMID:20069559

14. Plasencia W, Maiz N, Bonino S, Kaihura C, Nicolaides KH. Uterine artery Doppler at $11+0$ to $13+6$ weeks in the prediction of preeclampsia. Ultrasound Obstet Gynecol. 2007 Oct;30(5):742-9. https://doi.org/10.1002/uog.5157 PMID:17899573

15. Dahiana Marcela Gallo. Leona C. Poon, Ranjit Akolekara, Argyro Syngelakia KHN. Prediction of Preeclampsia by Uterine Artery Doppler at 20 - 24 Weeks'. Gestation. Fetal Diagn Ther. 2013;34:241-7. https://doi.org/10.1159/000356171.

16. Melchiorre K, Sutherland G, Sharma R, Nanni M, Thilaganathan $B$. Mid-gestational maternal cardiovascular profile in preterm and term pre-eclampsia: a prospective study. BJOG. 2013 Mar;120(4):496-504. https://doi.org/10.1111/1471-0528.12068 PMID:23190437

17. Kalafat E, Laoreti A, Khalil A, Da Silva Costa F, Thilaganathan B. Ophthalmic artery Doppler for prediction of pre-eclampsia: systematic review and meta-analysis. Ultrasound Obstet Gynecol. 2018 Jun;51(6):731-7. https://doi.org/10.1002/uog.19002 PMID:29330892

18. Sarno M, Wright A, Vieira N, Sapantzoglou I, Charakida M, Nicolaides $\mathrm{KH}$. Ophthalmic artery Doppler in prediction of preeclampsia at 35-37 weeks' gestation. Ultrasound Obstet Gynecol. 2020 Nov;56(5):717-24. https://doi.org/10.1002/uog.22184 PMID:32857890

19. Mucize Eric Ozdemir. Oya Demirci, Hatice Akay Ozturkmen, Nuray Bakal Ulusoy, Karolin Ohanoglu IUC. What Is the Role of the Maternal Ophthalmic and Cervical Internal Carotid Arteries in Predicting Maternal Adverse Outcomes in Preeclampsia. Am Inst Ultrasound Med; 2020. pp. 1-9.

20. Oliveira CA, Moreira de Sa RA, Zamprogno KV, Gutierrez da Matta F, do Vale Araújo F. Fabio Gutierrez da Matta F do VA. Magnesium sulfate and ophthalmic artery Doppler velocimetry in patients with severe preeclampsia : a case series. J Med Case Reports. 2017;11(1):1-6. https://doi.org/10.1186/s13256-0171490-1. 